\title{
The Impact of i-PUSH on Maternal and Child Health Outcomes, Health Care Utilization and Financial Protection: A Cluster Randomised Controlled Trial Based on Financial and Health Diaries Data
}

Amanuel Abajobir ( $\nabla$ aabajobir@aphrc.org )

African Population and Health Research Centre

Richard de Groot

Amsterdam Institute of Global Health and Development

Caroline Wainaina

African Population and Health Research Centre

Anne Njeri

African Population and Health Research Centre

Daniel Maina

African Population and Health Research Centre

Silvia Njoki

African Population and Health Research Centre

Nelson Mbaya

African Population and Health Research Centre

Hermann Pythagore Pierre Donfouet

African Population and Health Research Centre

Menno Pradhan

Vrije Universiteit Amsterdam

Wendy Janssens

Vrije Universiteit Amsterdam

Estelle M Sidze

African Population and Health Research Centre

Study protocol

Keywords: Maternal and child health, health care utilization, out-of-pocket health expenditures, health insurance, Universal Health Coverage, digital tools, Kenya

Posted Date: October 20th, 2020 
DOI: https://doi.org/10.21203/rs.3.rs-62882/v1

License: (c) (i) This work is licensed under a Creative Commons Attribution 4.0 International License. Read Full License 
The impact of $i$-PUSH on maternal and child health outcomes, health care utilization and financial protection: a cluster randomised controlled trial based on Financial and Health Diaries data

Amanuel Abajobir ${ }^{*}$, Richard de Groot $^{3}$, Caroline Wainaina ${ }^{1}$, Anne Njeri ${ }^{1}$, Daniel Maina ${ }^{1}$, Silvia Njoki ${ }^{1}$, Nelson Mbaya ${ }^{1}$, Hermann Pythagore Pierre Donfouet ${ }^{1}$, Menno Pradhan ${ }^{2,3}$, Wendy Janssens ${ }^{2,3}$, Estelle M Sidze ${ }^{1}$

${ }^{1}$ African Population and Health Research Centre, Nairobi, Kenya

${ }^{2}$ Vrije Universiteit Amsterdam, Amsterdam, Netherlands

${ }^{3}$ Amsterdam Institute of Global Health and Development, Netherlands

*Correspondence:

E-mail: aabajobir@aphrc.org

Mobile: +254 (0) 721141014 


\begin{abstract}
Background: Universal Health Coverage (UHC) ensures access to quality health services for all, with no financial hardship when accessing the needed services. Nevertheless, access to quality health services is marred by substantial resource shortages creating service delivery gaps in low-and middle-income countries (LMICs), including Kenya. The Innovative Partnership for Universal Sustainable Healthcare ( $i$-PUSH) program, developed by AMREF Health Africa and PharmAccess Foundation (PAF), aims to empower low-income women of reproductive age and their families through innovative digital tools. This study aims to evaluate the impact of $i$-PUSH on maternal and child health care utilization, women's health including their knowledge, behavior and uptake of respective services, as well as women's empowerment and financial protection. It also aims to evaluate the impact of the LEAP training tool on empowering and enhancing CHVs' health literacy and to evaluate the impact of the M-TIBA health wallet on savings for health and health insurance uptake.
\end{abstract}

Methods: This is a cluster randomised controlled trial (RCT) study that uses a four-pronged approach -including year-long weekly financial and health diaries interviews, baseline and endline surveys, a qualitative study and behavioral lab-in-the-field experiments-in Kakemega County, Kenya. In total, 240 households from 24 villages in Kakamega will be followed to capture their health, health knowledge, health-seeking behavior, health expenditures and enrolment in health insurance over time. A random half of the households live in villages assigned to the treatment group where $i$-PUSH will be implemented after the baseline, while the other half of the households live in control village where $i$-PUSH will not be implemented until after the endline. The study protocol was reviewed and approved by the AMREF Ethical and Scientific Review Board (AMREF-ESRC). Research permits were obtained from the National Commission for Science, Technology and Innovation (NACOSTI) agency of Kenya.

Discussion: People in LMICs often suffer from high out-of-pocket healthcare expenditures, which in turn, impedes access to quality health services. Saving for healthcare as well as enrolment in health insurance can improve access to healthcare by building capacities at all levels-individuals, families and communities. Notable, $i$-PUSH fosters savings for health care through the mobile-phone based "health wallet", it enhances enrolment in subsidized health insurance through the mobile platform-M-TIBA-developed by PAF, and it seeks to improve health knowledge and behavior through Community Health Volunteers (CHVs) who are trained using the LEAP tool-AMREF's mHealth platform. The findings will inform stakeholders to formulate better strategies to ensure access to UHC in general, and for a highly vulnerable segment of the population in particular, including low-income mothers and their children.

Keywords: Maternal and child health, health care utilization, out-of-pocket health expenditures, health insurance, Universal Health Coverage, digital tools, Kenya

Trial registration history: Registered with Protocol Registration and Results System (Protocol ID: AfricanPHRC; Trial ID: NCT04068571: AEARCTR-0006089; Date: 29 August 2019) and The American Economic Association's registry for randomised controlled trials (Trial ID: AEARCTR-0006089; Date: 26 June 2020). 


\section{Background}

There has been a renewed international commitment to Universal Health Coverage (UHC) aiming at ensuring all people have access to the health services they need without suffering from financial hardships. However, there are sustained resource shortages and service delivery gaps in many countries that prevent them from meeting the Sustainable Development Goal (SDG) 3.8 related to UHC (i.e., achieve universal health coverage, including financial risk protection, access to quality essential health-care services and access to safe, effective, quality and affordable essential medicines and vaccines for all). Achieving UHC is particularly important in achieving SGDs goals related to maternal and child health (i.e., reducing the global maternal mortality ratio to less than 70 per 100000 live births (SGD 3.1) and ending preventable deaths of newborns and children under 5 years of age (SGD 3.2). All countries are committed to reduce neonatal mortality to at least as low as 12 per 1000 live births and under5 mortality to at least as low as 25 per 1000 live births) by 2030. Evidence still shows that more than half of the world's population lacks access to health care of sufficient quality [1] and about 100 million people fall into extreme poverty each year due to ill-health [2], particularly in lowand middle-income countries. To translate commitment to UHC into a reality, still need to undertake systemic reforms that require strong management and organization.

Despite being classified as a middle-income country in 2014, Kenya still remains among the $25 \%$ poorest countries strongly affected by social and health inequality in the world $[1,2]$. More than one-third of Kenyans have an income below the poverty line (1.9 USD/day) [1-3]. Inequalities in access to healthcare, in particular maternal and child care are still rampant despite major improvements made through targeted policies over the past few years [4]. The poorest mothers are still far behind in terms of coverage of essential reproductive and maternal and child health services. The Government of Kenya has included UHC as one of its 'Big Four Agenda'-action points, which is anticipated to lead the transformation of the country by 2022 [3]. The objective is to achieve a $100 \%$ cost subsidy for essential health services and to reduce out-of-pocket health expenditures by half. Low-cost health insurance schemes, eHealth and mobile health (mHealth) services are among other opportunities to achieve this goal. Consequently, the non-governmental organisations AMREF Health Africa and PharmAccess Foundation (PAF) are both supporting the Government to achieve the goal of UHC in many counties. This includes Kakamega-a county in Western Kenya-where 
AMREF and PAF are jointly implementing their Innovative Partnership for Universal Sustainable Healthcare ( $i$-PUSH) program.

The $i$-PUSHprogram utilizes innovative digital tools developed by both partners to enhance access to affordable and quality health care to low-income women of reproductive age (WRA) and their families. Through the $i$-PUSH, women receive the National Health Insurance Fund (NHIF) SupaCover at subsidized premiums on their mobile phone, using the PAF's so-called "health wallet". The "health wallet" runs on the digital platform M-TIBA, which registers health care utilization at participating clinics, connecting patients, providers and clinics on one platform. The PAF is supporting the implementation of UHC by registering households using M-TIBA and developing a socio-economic mapping of the population. One such tool, Connected Diagnostics for malaria, was recently piloted in Kisumu County (Kenya). Another tool, designed to ease communication between doctors and women during pregnancy and postnatal period, has been piloted in Nairobi and there are plans to scale up in other counties. Community Health Volunteers (CHVs) are the first point-of-contact for women in the program. They make use of AMREF's Mjali (Mobile Jamii Afya Link) tool for digital registration of household information and the mobile phone-based LEAP tool for training. The LEAP tool employs a mobile learning approach to train and empower CHVs using their mobile devices operating from any phone [5]. This enables the CHVs to learn at their own pace, and with their own mobile devices while in the community, providing both interpersonal and community aspects of learning.

In order to maximize the effectiveness of this program, it is important for stakeholders to have a deep understanding of current access to health services, households' health-related decisionmaking and out-of-pocket health care expenditures in the target populations. The main aim of the study is to evaluate the impact of $i$-PUSH (most notably enhanced access to subsidized health insurance through the "health wallet" and increased CHV training opportunities through the LEAP training tool) on health care utilization, in particular related to maternal and child health, and financing of out-of-pocket health care expenditures. The evaluation research is conducted by the independent research institutes, namely, the African Population and Health Research Center (APHRC) and Amsterdam Institute for Global Health and Development (AIGHD). It is funded by the Dutch Postal Code Lottery, the Joep Lange Institute, and the Dutch Ministry of Foreign Affairs through the Health Insurance Fund. 


\section{Methods/Design}

\section{Study site}

$i$-PUSH has been ongoing in parts of Kakamega County since 2017. The study is being carried out in Khwisero Sub-county-one of the sub-counties in Kakamega, where the $i$-PUSH program will expand after the baseline survey. The implementing partners selected two health clinics that were under consideration to be included in the expansion of the $i$-PUSH program. Twentyfour (24) villages located in the catchment areas of these four clinics were randomly selected from a list of all villages in the catchment areas. The list of villages was provided by the Subcounty government jointly with the $i$-PUSH program area manager. Random selection was done by the research team using a computer program to generate a short list of villages from the longlist, to be randomly assigned to either the treatment or the control group. Villages cover on average about 100 households. Each village is served by one unique CHV.

\section{Study design and randomization procedure}

The survey design is a longitudinal cluster randomized controlled trial (RCT). Randomization occurs at the level of villages in Khwisero. The "treatment" and "control" groups are constructed, comparing villages where $i$-PUSH will be rolled out after the baseline with village where $i$-PUSH will not be rolled out until after the endline.

The research team used community-level socio-demographic and infrastructure indicators from baseline data to form pairs of similar villages and determine the exact matching indicators. In keeping with robustness of the cluster RCT, the procedure hence followed four steps for matching of the treatment" and "control" villages: (i) purposive selection of the Sub-county (Khwisero) where the intervention will roll out; (ii) random selection of 24 villages; (iii) pairmatching of villages based on relevant background characteristics and outcomes of interest; and (iv) randomization of treatment and control villages within each pair of the villages by flipping a coin. Pairing villages before randomization reduces the risk of a bad draw during the randomization process. Randomisation without pairing will, in expectation, also lead to similar control and treatment groups, but it is also possible that the random draw produces a control and treatment group with very different characteristics by chance [6]. This risk is reduced through pairing. We used the Euclidean distance for our matching process, which corresponds to the absolute difference between the standardized values of all of the covariates for a possible pair of matches. We conducted the matching within each of the four health clinic catchment areas. Thus, each village was matched with one of the other five villages in the vicinity of the health clinic. This was done to ensure that each health clinic had an equal number of treatment 
and control villages in their catchment area. Hence we computed this distance measure between each village and all other villages within the same health clinic catchment area; "pair" the two villages with the minimum distance and remove them from the list; repeat the distance calculation excluding the pair made; and continue until all villages were paired.

After the matching process, the randomization assignment was carried out in the presence of key stakeholders including PAF, local liaison persons and village representatives, upon explaining all steps. Consent for the procedures was obtained from local government officials before the random assignment. The following steps were followed: papers with paired village names were folded and put in a bag; and two village representatives from each paired village discussed on whom to pick the paper and after the other group members verified that the names could not be seen, one paper was picked. A Kenya Shilling 10 coin was used to decide which group the picked village belonged to by flipping the coin. The village representatives had decided that the head of the coin should represent the control group, justifying that Kenyatta ( $1^{\text {st }}$ president of Kenya) was a "controlling village", and the shield to represent the intervention group. The process of choosing the folded paper and flipping of the coin was repeated for all paired villages.

The treatment group thus consists of the target population living in the randomly assigned 12 villages. $i$-PUSH roll-out in the treatment villages includes training of their CHVs with the LEAP tool, who will subsequently introduce the health wallet to eligible women living in the treatment villages, and offer them the subsidized insurance scheme on their mobile phone. The $\mathrm{CHVs}$ working in the control villages (as well as the remaining non-sampled villages on the longlist) will not receive training on the LEAP tool yet, nor will women in the control villages be offered the health wallet and subsidized insurance on their mobile phone. They constitute our comparison group. We randomized at the village level because 1) villages are served by one CHV each, who are either trained or not trained on LEAP (hence, the LEAP intervention cannot be varied within villages); 2) to avoid contamination between households within the same villages regarding health-related knowledge and behavior; and 3) moreover, it was deemed politically unfeasible to offer the health wallet and subsidized health insurance to some eligible households in a village but not to other eligible households in that same village. Upon on roll out of the subsidized services, eligible households are encouraged to use the services, though they are given the right to opt out at any time. 


\section{Study population}

The study population consists of eligible households living in the selected study villages. Eligible households included those with at least one woman of reproductive age (WRA) (1849) who: a) had at least one child below 4 years living with her at baseline; or b) was pregnant at baseline.

Selected CHVs and PAF's area manager provided the list of households and other necessary information within the work area of each CHV. Based on the household demographics and pregnancy information, eligible households were identified. Initially, the study sought a 50-50 allocation between households with a pregnant woman and households with a child under 4 years old. After the household listing exercise, it became clear that there were too few pregnant women in each village to fulfill this criterion. We then decided to include all pregnant women in our sample, and randomly sample additional households with children under 4 years old until the cluster size (10 households per village) was achieved. The research team did a random selection as follows: all eligible households with children under 4 years old were entered in Excel sheet and receive a randomly assigned number. The team ordered households per CHV and 10 households per $\mathrm{CHV}$ in each village were included in the study sample. Additional eligible households per $\mathrm{CHV}$ were over sampled to serve as replacement households for dropouts.

\section{Sample size}

Sample size calculation followed Hemming et al. [7]'s study by fixing the number of clusters per arm to be 12 clusters, and then estimated the cluster size and total sample size. In the current study, it was assumed that the $i$-PUSH program could yield an effect size of 0.4 in terms of health care utilization with an intracluster correlation (ICC) of $\rho=0.014$. The estimates of the ICC were derived from Geng et al. [8]'s, study conducted in Nandi County which used highfrequency data on diaries on health-seeking behaviors and financial expenditures over one year (October 2012-October 2013). The calculation of the ICC was based on health care utilization measured as visits to any formal health provider, unconditional on reported health symptoms. It hypothesized a confidence interval of $95 \%$, a margin-of-error of $5 \%$ and a power of $80 \%$. The cluster size per arm, total women per clusters was therefore 10 and 12 women, respectively. Hence: 
$\mathrm{n}=2\left(\frac{\mathrm{Z}_{1-\frac{\alpha}{2}}+\mathrm{Z}_{1-\beta}}{\mathrm{ES}}\right)^{2}$, with ES (effect-size) the ratio between the mean difference and pooled standard deviation, $\mathrm{Z}_{1-\frac{\alpha}{2}}$ and $\mathrm{Z}_{1-\beta}$ are the values from the normal distribution holding $1-\frac{\alpha}{2}, 1-\beta$ below it, respectively. $\alpha$ and $\beta$ are levels of significance and power, respectively. Thus,

$n=2\left(\frac{1.96+0.84}{0.4}\right)^{2}=98$

By fixing the clusters per arms to $\mathrm{k}=12$, the required sample size per arms is given by the following formula:

$n_{C}=\frac{n k(1-\rho)}{k-n \rho}=\frac{98 \times 12 \times(1-0.014)}{12-(98 \times 0.014)}=109.10$

In the formula above, a feasibility check must be done so that $\mathrm{k}>\mathrm{n} \rho$. This condition is satisfied since $12>1.372$

If we assume an attrition rate of $10 \%$, then:

$n_{C}=\frac{109.10}{1-0.1} \approx 121$

To keep sample size at par, households that dropped out of the study before the start of the intervention were replaced with new eligible households on a rolling basis for a maximum period of six months, or until program start.

\section{Description of the $i$-PUSH program}

$i$-PUSH is a comprehensive intervention that ultimately aims to improve the utilization of Reproductive and Maternal and Child Health (RMNCH) services among WRA and their young children in Kakamega and Nairobi Counties, by increasing knowledge about and (financial) access to RMNCH services as well as improving the quality of care of RMNCH services. In the original $i$-PUSH program that is the focus of this evaluation, households receive the first year of health insurance premium for free, and they are stimulated and supported to save for a $50 \%$ co-payment in the second year and a $100 \%$ premium payment thereafter. The free provision in year one is expected to show the benefits of insurance to the selected households. The 50\% co-payment and the support for savings in the second year is expected to install a habit of savings. ${ }^{1}$

This evaluation study will focus on the first two of these three spheres of interest: knowledge and (financial) access; the third sphere of interest-quality upgrades at the health facilities,

\footnotetext{
${ }^{1}$ PAF is currently working on new approaches to differentiate the size of the co-payment by socioeconomic status, which could be more effective in targeting subsidized health insurance to those who cannot afford the full premium, while at the same time, introducing new incentives to save for co-payments for those who can afford them.
} 
cannot be evaluated with our study design because all $i$-PUSH clinics in our study area were already upgraded at baseline. A rough sketch to the implementation of enrolment, intervention and assessment of the programme is indicated in Annex 1.

\section{The LEAP training tool for CHVs}

Working with CHVs, $i$-PUSH will increase the knowledge on both RMNCH and on insurance/health financing among women and men in the target communities. To this end, $\mathrm{CHV}$ s receive additional training through the specially developed LEAP training tool carried out by AMREF. This tool contains modules on specific health terrains of interest (notably, health promotion activities for children under five, family planning, antenatal care, danger signs in pregnancy and after delivery, danger signs in children under five, maternal and child health and nutrition, water safety, hygiene and sanitation) as well as on health savings and health insurance. The CHVs can follow this training on the smartphone that they will receive as part of their inclusion in the program.

AMREF can currently evaluate if LEAP could improve the knowledge of its CHVs because at the end of each training module, $\mathrm{CHVs}$ can participate in a quiz on the tool that tests their newly gained knowledge or. We could then explore whether the improved knowledge translates into better knowledge and better health-related behavior among women and men in the target communities. Additionally, we could examine whether the LEAP training tool on top of the standard training activities of CHVs translate into improving women and men's knowledge and behavior on pre-specified topics. We will also assess whether CHVs' time spent on LEAP, number of training modules completed, and scores on the LEAP quizzes predict impact on women and men in the communities.

\section{Subsidized access to NHIF SupaCover health insurance through the M-TIBA health wallet}

The improved knowledge on health and health financing of women and men is also expected to translate into improved attitudes towards insurance and saving for health and insurance. To support these changes in knowledge and attitudes, WRA will receive the first year of their NHIF insurance premium at $100 \%$ subsidy and the second year at a 50\% subsidy. The subsidies are expected to enhance initial enrolment in NHIF such that enrollees can experience first-hand the benefits of insurance. Moreover, this will allow women to be acquainted with regular savings for the insurance premium for the next year, such that they will get into the habit of recurrent savings and increasingly be able to frequently set aside small amounts of money. 
An integral component of the $i$-PUSH program is the so-called "health wallet" on M-TIBA (a mobile payment platform). Most WRA in the target population have limited access to formal financial services such as bank savings accounts. The widespread availability of M-PESA opens a new avenue of change in this respect. To alleviate this constraint, $i$-PUSH offers women the opportunity to set aside money in a commitment savings device on their mobile phone. The only requirement is that the mobile number is registered on their personal name. This will allow them (and other people such as spouses, relatives) to transfer funds into the wallet through M-PESA, which are subsequently 'reserved' for direct payment of medical costs at M-TIBA-connected health providers or for future payment of the annual insurance copremium. This feature of the health wallet is expected to support women in their financial planning-funds transferred into the wallet are kept safe and secure until they are needed for health-related purposes. Additional small-scale interventions are added to the program to enhance further savings, such as the provision of a savings calendar. Thus, this component of the program expects to increase savings for health as well as uptake and renewal of health insurance among the target population.

Enrolment in health insurance is further facilitated through an additional feature of $i$-PUSHCHVs can digitally enrol WRA and their household members (spouses, children and other dependents) on the NHIF SupaCover as long as they have an ID or birth certificate available at the time of the $\mathrm{CHV}$ visit. The $\mathrm{CHV}$ uploads the required documents and takes care of the registration process on his or her smartphone (as provided by the $i$-PUSH program), saving the women from a lot of hassle in traveling back and forth to the insurance offices to hand over all the required documents and go through the administrative steps. In other words, $i$-PUSH is also expected to relieve logistical and time constraints to the uptake of insurance. Perhaps such a scheme enhances enrolment into the intervention or insurance [9].

\section{Quality upgrade of selected clinics}

Note that the $i$-PUSH program first identifies clinics for inclusion on the digital M-TIBA platform and for support with quality upgrade. This study will not evaluate the quality upgrade but only work with selected $i$-PUSH clinics. The catchment areas of this clinic are divided into villages that each include one $\mathrm{CHV}$ who is responsible for the village or approximately 100 households, dependent on the location. The program subsequently trains the CHVs in selected villages to provide $i$-PUSH insurance and services to the households on their list. Thus, the villages in the control group have access to the same upgraded clinics. However, households in control villages do not receive the subsidized health insurance, assistance in the 
administrative processes to enrol in health insurance and mobile phone-based health wallet that allows saving for health or receiving personalized text messages. Moreover, maternal and child health training of their CHVs is less comprehensive and only conducted during the monthly CHV meetings instead of complemented with the mobile phone-based LEAP training tool.

\section{Expected outcomes}

Overall, the outcomes of interest for this evaluation study include the following:

\section{Healthcare utilization}

- Healthcare utilization for curative care (conditional on illness and/or injury):

- Healthcare utilization for preventive care (immunization, growth monitoring children below 5 only).

- Continuum of maternal care (perinatal care - pregnant women only):

- Proportion of women who attended at least four times antenatal care during pregnancy.

- Proportion of women given folic acid/iron supplementation during pregnancy.

- Proportion of births attended by skilled birth attendant.

- Proportion of postnatal care visits after delivery.

\section{Health outcomes}

- Proportion of children malnourished (anthropometrics).

- Proportion of common childhood illnesses (e.g., diarrhoea, acute febrile illnesses, acute respiratory infections, etc.)

- Proportion of women self-reporting perinatal depression.

- Proportion of common mental illnesses in adults.

- Proportion of other illnesses (infectious, non-communicable diseases, etc.).

\section{Health knowledge and behavior}

- Proportion of knowledge, attitude and practice to maternal and child health

- Proportion of children with adequate feeding practices

○ Proportion of children meeting minimum meal frequency

- Proportion of children meeting minimum dietary diversity

- Proportion of children sleeping under a bed net

\section{Financial protection}

- Out-of-pocket healthcare expenditures

- Proportion of people enrolled in NHIF

- Proportion of people saving through M-TIBA 
- Total amount saved on M-TIBA

- Proportion of people on track with savings for insurance premium on M-TIBA

The study will provide additional insights into the following digital tools that are currently being rolled-out by AMREF or PAF:

\section{Data collection instruments and techniques}

Data collection consists of four main components: 1) a qualitative baseline study, 2) baseline and endline household surveys, 3) weekly financial and health diaries interviews with all adults and emancipated minors in the households, and 4) behavioral lab-in-the-field experiments. Both quantitative and qualitative tools were piloted in Nairobi slums and debriefed to the research team including the field team.

\section{Qualitative baseline study}

The study uses qualitative data collection methods to get a deeper understanding of the perceptions and behaviors of the population on health insurance and health care utilization, to feed into the quantitative instrument design and to complement findings from the quantitative surveys. This will help understand the motivations, drivers and obstacles to savings for insurance in the target population. The qualitative baseline study is based on in-depth interviews (IDIs, $\mathrm{n}=20$ ) and focus group discussions (FGD, $\mathrm{n}=4$ ) with different stakeholders who were purposively sampled after CHVs' mobilization to willingly participate in the study. Another qualitative study may be conducted after the quantitative endline survey has been completed to provide additional under-the-skin description and/or for improved interpretation if the impact evaluation yields unexpected results.

\section{Baseline and endline survey instruments}

The quantitative evaluation starts with a baseline survey before the rollout of the $i$-PUSH program and is completed by an endline survey conducted one year after the $i$-PUSH introduction. Both surveys include modules on household demographics, socio-economic indicators, food and non-food consumption indicators, financial inclusion, participation in community networks, as well as self-assessed health status, health-related knowledge and behavior, health care utilization and health expenditures, maternal health, mental health, intrahousehold decision-making processes and gender dynamics. In addition, the endline will include a satisfaction module on participation in the $i$-PUSH program for women in the treatment areas only. The household head or the most knowledgeable household member respondents to the household roster, household-level modules and questions about under-aged 
household members. The focal person for the modules on maternal health, intra-household decision-making and health expenditures is the WRA in the household. All remaining individual-level modules are reported by all adult individuals in the household. (Table 1).

Table 1: Modules covered in the study, unit of responses and respondents

\begin{tabular}{|c|c|c|}
\hline Modules & Unit of response & Respondent \\
\hline 1. Cover page & Household & Enumerator \\
\hline 2. Household roster & All household members & Head/most informed member \\
\hline 3. Socio-demographics & All household members & Head/most informed member \\
\hline 4. Education & $\begin{array}{l}\text { All household members }>5 \\
\text { years }\end{array}$ & Head/most informed member \\
\hline 5. Health outcomes & All household members & Head/most informed member \\
\hline 6. Health care utilization & All household members & Head/most informed member \\
\hline 7. Food consumption & Child $<4$ years & Head/most informed member \\
\hline 8. Housing and assets & Household & Head/most informed member \\
\hline 9. Employment and income & $\begin{array}{l}\text { All household members }>12 \\
\text { years }\end{array}$ & Head/most informed member \\
\hline 10. Participation in diaries & $\begin{array}{l}\text { All adults or emancipated } \\
\text { minor }\end{array}$ & Individual \\
\hline 11. Women's empowerment & $\begin{array}{l}\text { Female adult or emancipated } \\
\text { minor }\end{array}$ & Individual \\
\hline $\begin{array}{l}\text { 12. Preferred clinics and } \\
\text { mobile money }\end{array}$ & $\begin{array}{l}\text { All adults or emancipated } \\
\text { minor }\end{array}$ & Individual \\
\hline 13. Mental health & $\begin{array}{l}\text { All adults or emancipated } \\
\text { minor }\end{array}$ & Individual \\
\hline 14. Women's health & $\begin{array}{l}\text { Female adult or emancipated } \\
\text { minor }\end{array}$ & Individual \\
\hline $\begin{array}{l}\text { 15. Savings groups and } \\
\text { cooperatives }\end{array}$ & $\begin{array}{l}\text { All adults or emancipated } \\
\text { minor }\end{array}$ & Individual \\
\hline 16. Financial instruments & $\begin{array}{l}\text { All adults or emancipated } \\
\text { minor }\end{array}$ & Individual \\
\hline 17. Health knowledge & $\begin{array}{l}\text { Female adult or emancipated } \\
\text { minor }\end{array}$ & Individual \\
\hline
\end{tabular}

\section{Financial and health diaries}

This study uses financial and health diaries data collection methodology as its core research instrument, because this technique is well suited to address the research objectives by providing a granular insight into the financial lives and health-related decisions of participating households. The financial diaries record all financial transactions such as purchases, gifts, remittances and loans, including those between household members in the seven days prior to each interview. Transactions are described by type, incoming or outgoing cash, amount, 
purpose, transaction partner and date. The health diaries provide a detailed picture of the incidence of illnesses and injuries, as well as preventive and curative health-seeking behavior. Health diaries collect data on all health events that occurred to any of the household members (respondents, their children and other household members) in the seven days prior to each interview. This includes symptoms, whether any health care was sought, which health provider was visited, health services received, out-of-pocket health expenditures, date of onset of the symptoms and date of provider visit(s).

Respondents to the weekly diaries interviews are all adults in the study households who are economically active (i.e. who handle money, excluding young adults who are still studying) and capable of conducting the interview (i.e. excluding very old-age or disabled household members). Emancipated minors, i.e. teenage girls who are a mother or who are pregnant, are also included as diaries respondent. The financial diaries are reported by each individual himor herself. The health diaries can be reported by an individual for the entire household.

Data are collected through digital tools that allow to analyze data as they are recorded, and improve data collection tools mid-course of the process. The short recall period drastically reduces recall bias and ensures that both major and minor illness episodes, including those with foregone care are reported. Because interviewers visit households weekly, they build a relationship of trust, which enables the diaries to capture also more sensitive health events. This is of particular importance in relation to capturing maternal and child health experiences. Moreover, in contrast to what is common in standard RCT, the shorter research cycles, and report results on a regular basis to allow program managers to learn and take action for continuous improvement.

Qualified fieldworkers were recruited and trained on data collection tools and techniques. Diaries data are collected through personal interviews in a conversation-like manner. Interviewers record information navigating through a specialized software program while the interview unfolds. Prior experience of the research team with similar data collection indicates that such interviews generally last 10-15 minutes as both interviewers and respondents get more experienced. To reduce burden on the respondents, interviewers take care in planning the day, time and location of the weekly interviews at the convenience of the respondent. The respondents are interviewed separately and in private spaces to ensure confidentiality. The COVID-19 situation has switched the mode of data collection to telephone interviews. 
Fieldworkers were retrained on telephone interview ethics and techniques. This will be implemented until the crisis recedes.

At regular intervals, the weekly diaries are complemented with pop-up modules. In particular, these include on a monthly basis: a pregnancy module, perinatal depression, and general mental health; and on a quarterly basis: food consumption of children, anthropometrics of children and their mothers. Since the COVID19- outbreak, a monthly module has been added to assess the effect of COVID-19 pandemic on COVID19-related knowledge, preventive behavior (such as hand-washing and social distancing), mobility, health-seeking behavior, and fear/worries.

\section{Behavioral lab-in-the-field experiments}

Diaries respondents are also invited to participate in a number of incentivized behavioral labin-the-field games to measure women's empowerment, risk attitudes [10], and time preferences. As is standard in the behavioral economics literature, respondents can earn (small) amounts of money dependent on their decisions. This has been shown to improve accuracy of responses. The research team has extensive experience in conducting similar games in Kenya, Tanzania, Nigeria and elsewhere. All payments are made directly to the respondent through MPESA.

\section{Data management and analysis}

Trained team leaders are situated in the field to supervise real time data collection. Data quality is ensured by regular spot checks and sit-ins to approximately $5-10 \%$ of each fieldworker's daily work to verify authenticity of the data collected. Data collection is done electronically using tablets/phones, with spot checks for quality control. The field supervisors certify the quality of the data through editing the data before they are transferred to the database. Once the data collection is completed and synchronized in centrally located database, all inconsistencies are resolved prior to data analysis. An automated routine to check on the data completeness, correctness and consistency runs on $100 \%$ of the collected data. A discrepancy report is generated to help in following up on any inconsistencies or errors in the data with the responsible interviewer. Similarly, the quality of qualitative data will be ensured through recruitment and training of qualified field interviewers with experience in qualitative data collection. A qualified transcriber will transcribe the interviews verbatim and double coding of about $10 \%$ of the transcripts will also be done to ensure consistency in the application of the codes. Access to data is granted for all research teams in respective research organizations. 
Data auditing is carried out by the research team on a progressive basis on weekly basis throughout the study duration.

Quantitative data will be analyzed using Stata version 14 statistical software. The first set of analysis will consist of descriptive statistics and will summarize and compare using measures of central tendency and dispersion (mean (SD), range and median). This will allow us to detect similarities and/or differences between participants' characteristics across the different subgroups. In other words, we will compare some baseline measurements between the control and intervention groups using t-test adjusted for clustering at the village unit for continuous variables, and cluster-adjusted chi-square for binary variables. We will first check whether the outcomes and covariates in the control group and treatment group are comparable at baseline. The second set of analysis will consist assessing the causal effect of the $i$-PUSH intervention via an analysis of covariance (ANCOVA) based on intention-to-treat analysis (all respondents who are randomized will be included in the statistical analysis and will be analyzed according to the group they were initially/originally assigned). Based on our research questions, we will explore several econometric models as outlined in Appendix 2. Interim data analyses are done using baseline and weekly diaries data.

\section{Risks and measures to minimize them}

As the information collected will be on health service delivery, financial and insurance information, we do not anticipate any risks to participants, nevertheless. We will aim to minimize the risks by being as forthcoming as possible on the project description and the ethical process. In the case of minimal discomfort as a result of personal and sensitive questions, the research team will endeavor to ensure that the participant is given ample time to compose themselves, reassure them of confidentiality and ability to stop the interview if they are not able to continue with the interview. Ancillary and post-trial care may not be indicated as this is a community trial that does not involve any significant harm to the participating households.

\section{Dissemination of findings}

Scientific dissemination through peer-reviewed publications and implementing partners' dissemination through quarterly updates (e.g., meetings, presentations and brainstorm sessions) will ensure that findings are shared on a regular basis. These activities allow both parties to benefit from each other's insights and interpretations. Annual PharmAccess strategy meetings facilitate sharing knowledge with all country offices. Moreover, APHRC has good links with Kenyan policy makers in the field of health, and PAF is well connected to national and countylevel government officials, the NHIF, as well as many local healthcare providers. Finally, the 
linkages between the research group, PAF and the Dutch Ministry of Foreign Affairs with respect to health financing ensure regular sharing of findings at the Dutch national policy level. The close connection of the AIGHD with the Joep Lange Institute and the Amsterdam Technology and Health Institute (ATHI) facilitates sharing of findings with parties active in social technologies (start-ups working on the interface of digital technologies and health, mobile payment platforms).

\section{Ethical considerations}

The protocol was reviewed and approved by APHRC's internal ethical review committee and AMREF's accredited ethical review board. Ethical clearance was obtained on 21 April 2020 with number P679-2019. Research permits were obtained from the National Commission for Science, Technology and Innovation (NACOSTI) agency of Kenya. Informed consent from study participants was obtained upon detail orientation on project information, the purpose of the research study, possible risks and benefits. Model consent form is provided in separate file. All interviews are conducted in privacy and no identifying information is included in any data or reports; all data is anonymized to protect the identity of respondents. The participants are given a unique code and all identification data of participants shall be shredded/destroyed after analysis has been completed. Further ethical approval will be sought for any modifications to the protocol which may impact on the conduct of the study.

\section{Discussions}

Despite various international efforts to improve maternal and child health globally, several low and middle-income countries (LMICs) especially those in sub-Saharan African (SSA) countries are struggling with low rates of maternal and child survival. In Kenya, the maternal mortality ratio has marginally reduced to 362 per 100,000 live births, not statistically different from the figures reported in 2008-2009 [11]. While a substantial under five mortality reduction was achieved with a drop from 115/1000 live births in 2003 to 52 per 1000 live births in 2014, it is still two-fold higher than the SDG target [11]. These figures are disproportionately higher in Western Kenya, such as in Kakamega.

Improving access to health care throughout pregnancy, childbirth and during childhood is key in improving maternal and child health. Experience over the past decade has shown that building capacities of individuals, families and communities to ensure appropriate self-care, prevention and care-seeking behavior improves maternal and child health outcomes [12]. However, this is more difficult to achieve in poor populations who have worse health outcomes 
than the non-poor. Barriers such as costs of care, lack of information and cultural beliefs impede access to health care among poor communities.

Since its independence in 1963, the government of Kenya has initiated policy, reforms and strategies towards UHC for all, including those in vulnerable situations such as low-income mothers and children. In 1998, the NHIF act was amended to enhance coverage among the poor, accelerate coverage of the informal sector, and enhance the benefit package $[13,14]$. The most recent reform along the same line was the introduction of free maternal health services in 2013 that included abolition of user fees at primary health care facilities [14]. Despite these positive steps, Kenya's implementation of UHC has been riddled with myriads of challenges including poor quality of care, utilization and catastrophic spending by households especially the poor and other vulnerable groups [16]. Overall, health insurance coverage in Kenya has only increased from $8 \%$ to $20 \%$ between 2009 and 2014, and those from wealthy households were 12 times more likely to have insurance compared to those in poor households. Similarly, those in the informal employment and rural settings were less likely to be insured [11].

The $i$-PUSH program thus has been initiated to accelerate the expansion of health insurance coverage among the low-income population, WRA and their family members, using innovative digital tools. This research investigates the reasons for low access to health services, in particular related to maternal and child services, where the problems are persistent. It also investigates in-depth to what extent costs of services hinder access, and whether expanding UHC through the $i$-PUSH program is an effective strategy to increase access. Information generated from this study will be instrumental in improved implementation of policies supporting the roll-out of UHC. This research will also provide valuable information on UHC policies for the academic community, in particular, because we use high-frequency data (diaries) and analyze digital technologies that can support UHC.

To achieve these objectives, the study assumes the following: (i) no contamination across treatment and control groups; this is enhanced by a focus on villages and CHVs across villages, instead of individual women; (ii) County (Sub-county), AMREF and PAF will stick to the randomization plan that was independently developed by the research team but in agreement with stakeholders, and a memorandum of understanding (MoU) was signed among the partners; and (iii) Government does not unexpectedly and drastically alter its UHC policy plans in Kakamega (i.e., the government does not suddenly decide to offer free public care in Kakamega County, because that will take away many of the benefits of $i$-PUSH). Although 
there is little the research team can do in that case, this is the reason a flexible, high-frequency diaries design instead of standard (less flexible) RCT design is chosen. That is, there are more data points and if policies change half way, it is possible to actually capture this in the data.

This project is not free from limitations. One of the main limitations of the study is related to the exclusion of the following components in the impact evaluation, though they are an integral part of $i$-PUSH program. These include four components:

(i) Capacity-building at the regional level: $i$-PUSH invests in improving the capacity of community-based organizations to increase community-wide dialogue on RMNCH,

(ii) Health facility quality upgrades: the improved capacity of healthcare providers (implemented through $\underline{\text { SafeCare) }}$ to deliver RMNCH services is expected to lead to improved standards of care, improved quality of services and enhanced client satisfaction.

(iii) M-JALI household registration tool: AMREF has developed a digital tool to increase the capacity of CHVs to keep a census of the households in their target area, and collect household survey data on a rolling basis that can be used for the systematic reporting of community-level data, thereby potentially enhancing health-related decision-making and resource allocation at all policy levels.

(iv) M-TIBA digital health platform: PAF aims to increase the capacity of healthcare workers on learning, data capturing and reporting through the M-TIBA platform that allows amongst others for digital recording of health visits, diagnoses, treatments and services as well as payments/billing between patients, NHIF, Ministry of Health and providers.

These components are left out of the evaluation because our study focuses on the demandside (target population), and the described components are implemented either at the community-level or at the facility-level.

\section{Conclusions}

This study aims to evaluate the impact of $i$-PUSH program on maternal and child health care utilisation, women's health including their knowledge, behaviour and uptake of respective services, as well as women's empowerment and financial protection. It also aims to evaluate the impact of the LEAP training tool on empowering and enhancing CHVs' health literacy, and to evaluate the impact of the M-TIBA health wallet on savings for health and health insurance uptake. The findings will inform stakeholders to formulate better strategies to ensure access to 
UHC in general, and for those highly vulnerable segments of the population in particular. The findings of this research will provide valuable information on UHC policies for the academic community, policy-makers and other stakeholders to support the achievement of SDGs.

\section{Trail status:}

Protocol was registered in NIH - ClinicalTrials.org with registration number: AfricanPHRC; Trial ID: NCT04068571 dated on 28 August 2019 (https://clinicaltrials.gov/ct2/show/NCT04068571). Protocol amendment number: 01. It was also registered in The American Economic Association's registry for randomized controlled trials (Trial ID: AEARCTR-0006089) dated on 26 June 2020 (https://www.socialscienceregistry.org/trials/6089). Recruitment began in November 2019 and will continue until June 2021.

List of abbreviations/acronyms:

AIGHD - Amsterdam Institute for Global Health and Development

AMREF - African Medical and Research Foundation

ANC - antenatal care

APHRC - African Population and Health Research Center

CHV - Community Health Volunteers

FGD - Focus Group Discussion

ICC - Intracluster Correlation

IDI - In-depth Interview

$i$-PUSH - Innovative Partnership for Universal Sustainable Healthcare

LMICs - Low and Middle-income Countries

KDHS - Kenya Demographic and Health Survey

NACOSTI - National Commission for Science, Technology and Innovation

PAF - PharmAccess Foundation

PNC - Postnatal Care

RCT - Cluster Randomized Controlled Trial

SSA - sub-Saharan African 
WRA - Women of Reproductive Age

UHC - Universal Health Coverage

\section{Declarations}

Ethical approval and consent to participate: Obtained (reference number: P679-2019). Informed consent was obtained from all study participants.

Consent to publication: Not applicable.

Availability of data and materials: Data will be stored securely at the APHRC and Vrije Universiteit data repositories and available upon reasonable request after publication.

Competing interest: The authors declare that they have no competing interests

Funding: The data collection and research are funded by the Dutch National Postcode Lottery, the Joep Lange Institute, and the Dutch Ministry of Foreign Affairs through the Health Insurance Fund. The funder does not have any role in the design of the study and collection, analysis, and interpretation of data and in writing the manuscript.

Acknowledgement: The research team is highly grateful for the funder, study participants and the field team as well as all stakeholders directly and indirectly contributing for the smooth flow of the study.

Authors' Contributions: EMS, WJ, MP and HPPD conceived the study and its design; AA, RG and CW implement the study; AN manages the data; DM, SN and NM developed software for the implementation of the study.

\section{References}

[1] WHO. Tracking Universal Health Coverage: First Global Monitoring Report - World Health Organization - Google Books. 2015.

[2] Wagstaff A, Flores G, Hsu J, Smitz MF, Chepynoga K, Buisman LR, van Wilgenburg K, Eozenou P. Progress on catastrophic health spending in 133 countries: a retrospective observational study. Lancet. Glob. Heal.6:2018; e169-e179.

[3] Wangui E, Kandie C. Refocusing on quality of care and increasing demand for services: Essential elements in attaining universal health coverage in Kenya. 2016.

[4] Wehrmeister FC, Fayé CM, da Silva ICM, Amouzou A, Ferreira LZ, Jiwani SS, et al. Wealth-related inequalities in the coverage of reproductive, maternal, newborn and child health interventions in 36 countries in the African Region. Bulletin WHO. 2020; 98:394405

[5] AMREF Health Africa.Leap, the mHealth platform [online].

[6] Imai K, King G, Nall C. The essential role of pair matching in cluster-randomized experiments, with application to the Mexican universal health insurance evaluation. Statistical Science. 2009; 24: 29-53. 
[7] Hemming K, Girling AJ, Sitch AJ, Marsh J, Lilford RJ. Sample size calculations for cluster randomised controlled trials with a fixed number of clusters. BMC Med. Res. Methodol. 2011; 11.

[8] Geng X, Janssens W, Kramer B, van der List M. Health insurance, a friend in need? Impacts of formal insurance and crowding out of informal insurance. World Dev. 2018; 111:196-210.

[9] Banerjee A, Finkelstein A, Hanna R, Olken BA, Ornaghi A, Sumarto S. The challenges of universal health insurance in developing countries: Evidence from a large-scale randomized experiment in Indonesia (No. w26204). National Bureau of Economic Research. 2019.

[10] Andreoni J Sprenger C. Estimating time preferences from convex budgets," Am. Econ. Rev.2012; 102:3333-3356.

[11] KDHS. Kenya demographic and health survey: Key indicators. 2014; 1-76,

[12] United Nations Department of Economic and Social Affairs. SDGs: Sustainable development knowledge platform," United Nations. 2015.

[13] Kerber KJ, de Graft-Johnson JE, Bhutta ZA, Okong P, Starrs A, Lawn JE. Continuum of care for maternal, newborn, and child health: from slogan to service delivery. Lancet. 2007; 370:1358-1369.

[14] Okech TC, Lelegwe SL. Analysis of universal health coverage and equity on health care in Kenya. Glob. J. Health Sci. 2015; 8:218

[15] Mulupi S, Kirigia D, Chuma J. Community perceptions of health insurance and their preferred design features: Implications for the design of universal health coverage reforms in Kenya. BMC Health Serv. Res. 2013; 13.

[16] Calhoun LM, Speizer IS, Guilkey D, Bukusi E. The Effect of the removal of user fees for delivery at public health facilities on institutional delivery in urban Kenya," Matern. Child Health J. 2018; 22:409-418.

Appendix 1: Schedule of enrolment, interventions, and assessments

\section{Study period}

\begin{tabular}{cccc}
\hline $\begin{array}{c}\text { Enrolmen } \\
\mathbf{t}\end{array}$ & Allocation & $\begin{array}{c}\text { Post- } \\
\text { allocation }\end{array}$ & Close-out \\
\hline $\begin{array}{c}\text { November } \\
2019- \\
\text { June 2020 }\end{array}$ & $\begin{array}{c}\text { July - } \\
\text { September } \\
2020\end{array}$ & July 2021 & July 2021 \\
\hline
\end{tabular}

\section{Enrolment:}

Eligibility screen $\quad X$

Informed consent $\quad \mathrm{X}$

List other

procedures $\mathrm{X}$ 


\begin{tabular}{|c|c|c|c|c|}
\hline Allocation & & $X$ & & \\
\hline \multicolumn{5}{|l|}{ Interventions: } \\
\hline Intervention & $X$ & $\mathrm{X}$ & $\mathrm{X}$ & $X$ \\
\hline Control & $X$ & $\mathrm{X}$ & $\mathrm{X}$ & $\mathrm{X}$ \\
\hline \multicolumn{5}{|l|}{ Assessments: } \\
\hline $\begin{array}{r}\text { List baseline } \\
\text { variables }\end{array}$ & $\mathrm{X}$ & & & \\
\hline $\begin{array}{r}\text { List outcome } \\
\text { variables }\end{array}$ & $\mathrm{X}$ & & & $\mathrm{X}$ \\
\hline $\begin{array}{r}\text { List other data } \\
\text { variables }\end{array}$ & $\mathrm{X}$ & & & $\mathrm{X}$ \\
\hline
\end{tabular}

\section{Appendix 2: Econometric models}

\section{Impact of the $i$-PUSH on health and financial outcomes}

We will explore the impact of the $i$-PUSH program on health outcomes (health status, healthcare utilization, percentage of children who are fully immunized, etc.) and financial outcomes (percentage of women using NHIF/MTIBA to access services, percentage of people enrolled in NHIF through M-TIBA, percentage of people saving through M-TIBA, total amount saved on M-TIBA, etc.) via an analysis of covariance (ANCOVA) based on intentionto-treat (ITT) analysis. Since we will have several rounds of data collection on the same respondents, we will combine all rounds of data collection (baseline and follow-up waves) and we will run an ANCOVA econometric model on a pooled panel data. More specifically, in the econometric model below, we will regress the follow-up measurement of the outcome variable (dependent variable) on the following covariates: baseline measurement (pretest measurement) and treatment group while controlling for the baseline socio-demographic characteristics such as maternal age, education, socioeconomic status (wealth quintile), crowding (persons per room), occupation, etc. The considered model is thus:

$Y_{i j}=\alpha_{0}+\alpha_{1} X_{i j 0}+\alpha_{2}$ Treament $_{i j}+e_{i j t}$, 
with $Y_{i j}, X_{i j 0}$ the posttest and pretest/baseline measures of the outcome for the $i^{\text {th }}$ respondent from the $\mathrm{j}^{\text {th }}$ cluster, respectively. Treament ${ }_{i j}$ is a dummy for being assigned to the treatment group. It takes one if the respondent belongs to the intervention cluster and zero if control. $\alpha_{2}$ is the ITT effect or the impact of the $i$-PUSH program. For continuous outcomes, we will use an OLS model with standard errors clustered at the level of the community units. For binary outcomes, we will use a logit model. $e_{i j t}$ is the error term.

In addition to pretest measures, all other baseline covariates such as age, education, socioeconomic status (wealth quintile), crowding (persons per room), occupation, etc. will also be included in Equation (1). Thus, in Equation (1) the treatment effect of the I-PSUH program $\alpha_{2}$ assesses the treatment difference on post-treatment outcome adjusted for baseline.

\section{Impact of the $i$-PSUH on women empowerment}

We will assess whether the $i$-PUSH will significantly contribute in empowering women. For self-reported surveys on women empowerment, we will construct a total score $^{2}$ of women empowerment which is the sum of each domain when the woman indicates that she has sole or joint decision-making power within the household (for each domain, the decision-making binary indicator will be equal to 1 if the woman respondent makes the decision alone or jointly with her partner and 0 otherwise). This total score will therefore be used as the dependent variable and Equation (1) above will be used to estimate the impact of $i$-PUSH program on women empowerment. Furthermore, with regards to the field experiment, we will follow Almås et al. (2018). In other words, we will still use the same econometric model (Equation 1) after constructing the dependent variable emanating from the field experiment. This dependent variable will be the willingness-to-pay ${ }^{3}$ which is the share that the woman is willing to pay when the experiment stops.

\footnotetext{
${ }^{2}$ A robustness check will also be done using a principal component analysis (PCA). Hence, an index of women empowerment will be constructed using the PCA.

${ }^{3}$ If the stakes offered to the husband is Khs. 400 and the last amount offered to the woman is Khs. 100, then the WTP is 0.75 (400-100)/400).
} 


\section{Supplementary Files}

This is a list of supplementary files associated with this preprint. Click to download.

- SPIRITChecklist.pdf 